\title{
Calibrating SECCM measurements by means of a nanoelectrode ruler. The intrinsic oxygen reduction activity of PtNi catalyst nanoparticles
}

\author{
Emmanuel Batsa Tetteh ${ }^{1, \S}$, Tobias Löffler ${ }^{1, \S}$, Tsvetan Tarnev ${ }^{1}$, Thomas Quast ${ }^{1}$, Patrick Wilde ${ }^{1}$, Harshitha \\ Barike Aiyappa ${ }^{1}$, Simon Schumacher ${ }^{2}$, Corina Andronescu ${ }^{2}$, Richard D. Tilley ${ }^{3}$, Xingxing Chen $^{4}(\bowtie)$, Wolfgang \\ Schuhmann $^{1}(\square)$ \\ ${ }^{1}$ Analytical Chemistry-Center for Electrochemical Sciences, Faculty of Chemistry and Biochemistry, Ruhr University Bochum, Universitätsstr. \\ 150, D-44780 Bochum, Germany \\ ${ }^{2}$ Chemical Technology III, Faculty of Chemistry and CENIDE-Center for Nanointegration, University Duisburg Essen, Carl Benz Str. 199, \\ D-47057 Duisburg, Germany \\ ${ }^{3}$ School of Chemistry and Australian Centre for NanoMedicine, University of New South Wales, Sydney 2052, Australia \\ ${ }^{4}$ Research Group of Functional Materials for Electrochemical Energy Conversion, School of Chemical Engineering, University of Science and \\ Technology Liaoning, 114051 Anshan, China \\ ${ }^{\S}$ Emmanuel Batsa Tetteh and Tobias Löffler contributed equally to this work.
}

(C) The Author(s) 2021

Received: 18 April 2021 / Revised: 19 June 2021 / Accepted: 20 June 2021

\begin{abstract}
Scanning electrochemical cell microscopy (SECCM) is increasingly applied to determine the intrinsic catalytic activity of single electrocatalyst particle. This is especially feasible if the catalyst nanoparticles are large enough that they can be found and counted in post-SECCM scanning electron microscopy images. Evidently, this becomes impossible for very small nanoparticles and hence, a catalytic current measured in one landing zone of the SECCM droplet cannot be correlated to the exact number of catalyst particles. We show, that by introducing a ruler method employing a carbon nanoelectrode decorated with a countable number of the same catalyst particles from which the catalytic activity can be determined, the activity determined using SECCM from many spots can be converted in the intrinsic catalytic activity of a certain number of catalyst nanoparticles.
\end{abstract}

\section{KEYWORDS}

intrinsic electrocatalytic activity, nanoelectrochemistry, scanning electrochemical cell microscopy (SECCM), PtNi nanoparticles, single entity electrochemistry, alkaline medium

\section{Introduction}

The performance of an electrocatalyst can be generally augmented either by quantitatively increasing the density of active sites through optimisation of the film thickness and their availability through enhanced porosity [1-5] or by means of improving their intrinsic activity through judicious tailoring of the active sites according to the specified structure-property correlation. Since the former approach has its architectural limitations, recent advancement in catalyst development has witnessed a lookout for efficient structures based on theoretical calculations or modelling [6-8]. Although utilisation of these tools allows deeper fundamental understanding of structureactivity correlations leading to predictions of improved catalysts $[9,10]$, a practical verification of such structures unequivocally requires access to experimental methods capable of revealing the intrinsic activity of the catalyst, isolated from all matrix effects. Typically, the total apparent catalyst activity includes contributions from variations in the electrode coverage, film thickness, or particle size, which can be compensated by normalizing the activity with respect to the electrochemically accessible surface area [11, 12]. This approach marks a step closer towards examining the "real" catalyst properties as they consider the electrochemically viable catalyst surface area instead of the geometric electrode area. However, since the obtained catalytic activity is still influenced by film properties such as conductivity, film interactions, and surface coverage of additives, it cannot be used to derive the intrinsic activity of the catalyst. Ideally, the inherent activity of any catalyst nanoparticle (NP) can only be analysed in the absence of the aforementioned matrix effects. In this regard, multiple approaches are reported that aim to fulfil this requirement [13-20].

More specifically, measuring the intrinsic activity requires i) the absence of any matrix effects by analysis of isolated NPs (and barring electrode support interactions), ii) the detection of the catalytic current, which can be solely attributed to the catalyst NPs (excluding the current contribution of the underlying electrode surface), iii) the normalisation by the electrochemically active surface area of the NPs representing the number of active sites on the surface, which contribute to the catalytic current. 
Scanning electrochemical cell microscopy (SECCM) [21, 22] is one of the proposed techniques, which provides several advantages. It excels in combining a high spatial resolution, allowing to unveil local activity inhomogeneities of planar substrates, with statistical data due to automatized scanning or hopping along surfaces providing information from a large number of surface locations [23]. It was mainly employed in the analysis of local activity differences (steps/edges, grain boundaries) of bulk materials [24-29] or in the deposition of polymers [30]. Through combination with characterisation techniques, it was possible to provide fundamental kinetic information, making it a powerful tool for revealing intrinsic properties [31, 32]. NPs have already been investigated with SECCM by measuring the currents during experiments involving collisions [33] or landing [34] of NPs onto a surface from the suspension within the SECCM capillary. The activity of NPs already pre-deposited on a surface has also been visualised either directly [17] or with the help of an optical system to locate individual NPs prior to SECCM measurements [35]. Moreover, by using carbon-coated transmission electron microscopy (TEM) grids as a substrate for SECCM measurements, smaller nanoparticles including a few atom clusters were studied with post-electrochemical TEM and scanning TEM (STEM) analysis [36, 37]. Since the glass capillary does not make a physical contact with the substrate during SECCM measurements, there is enhanced mass transport of gaseous educts to reactive sites as well as faster product exchange with the surrounding environment. Hence, SECCM with gaseous educts or products is mimicking to a certain extent a gas diffusion electrode by creating a 3-phase gas/electrolyte/catalyst boundary [38]. The SECCM technique has been constantly refined since its introduction in order to allow its use in special applications such as non-aqueous electrolytes [39], photo-electrochemistry [40], and oil-coated substrates [41, 42].

Since the scanned surfaces are generally too large to be introduced into TEM, post-SECCM imaging is usually only possible for catalyst materials big enough to be seen in the scanning electron microscope (SEM). For catalysts with sizes generally relevant for catalytic applications in the range of 1-50 nm, quantification of the number of scanned particles is highly challenging. Hence the question arises, as to what information does an SECCM measurement provide for such samples. Is it still the intrinsic activity, which is being measured? What is a typical number of particles to be measured with typical currents? Is it a high number and thus, representing a statistically averaged information about the particle size distribution, or is each spot accidentally representing a different particle size distribution? Against this background, which conclusions can be drawn from the data? In this work, we aim to answer these questions by careful data analysis and utilisation of a complementary specifically designed ruler-type technique.

\section{Results and discussion}

In order to fulfil the requirements of intrinsic activity measurements using SECCM (Fig. 1), an electrode material of very low activity ("inert") is necessary. Therefore, boron doped diamond (BDD) [43] was chosen as underlying electrode material. The BDD electrode had a doping level between 500 and 1,000 ppm. The electrode is a $500 \mu \mathrm{m}$ disk attached to a conductive rod in brass and polished to an average roughness $\left(R_{\mathrm{a}}\right)<10 \mathrm{~nm} . \mathrm{PtNi}_{2} \mathrm{NPs}$ were used as catalyst, which are a well-known model material with high activity for the oxygen reduction reaction (ORR).

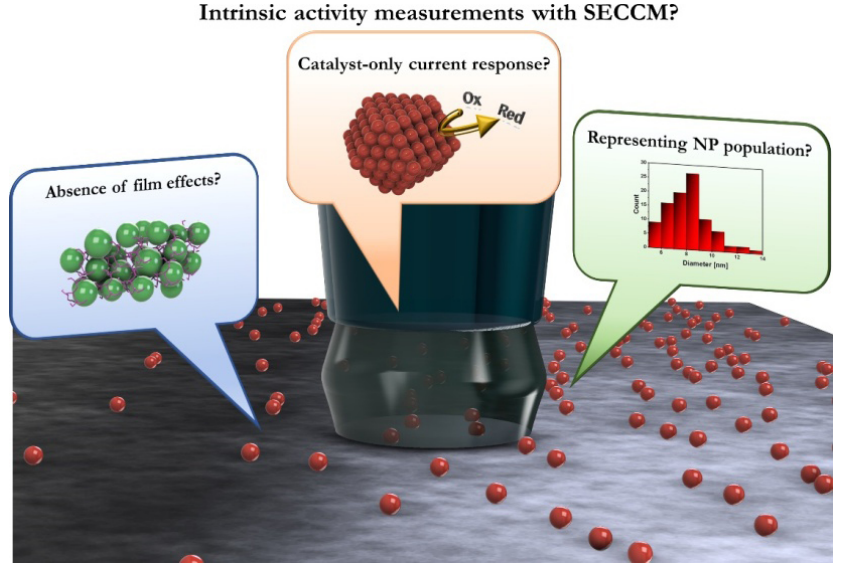

Figure 1 Factors, which must be considered for an SECCM measurement when information about intrinsic electrocatalytic activity is aspired.

Following a standard synthesis protocol [44, 45], NPs of about $9 \mathrm{~nm}$ in diameter and narrow size distribution were obtained, which were analysed by means of TEM (see Fig. S1 in the ESM). In a first step, the blank BDD electrode was investigated using voltammetric scans to record its contribution to the ORR current, which has to be considered due to the supposed sub-monolayer NP coverage. The current response only showed a marginal deviation between the 121 spots, confirming a consistent blank electrode linear sweep voltammogram (LSV), which can be used for electrode current contribution subtraction (Fig. 2(a)). After immobilisation of $\mathrm{PtNi}_{2} \mathrm{NPs}$ and removal of the oleylamine capping upon heat treatment at $200{ }^{\circ} \mathrm{C}$ for $2 \mathrm{~h}$ under ambient air, the measurement was repeated with the same electrode. The current at each spot was significantly higher due to the ORR current from the NPs, but the deviation between the spots was also much higher, which can be attributed to the different numbers of NPs at each measured location, i.e., the landing zone of the SECCM capillary during scanning. Current colour plots show that there is no trend between the LSVs and experimental artefacts can be excluded (Figs. 2(b) and 2(c)). We further recorded the potential of the quasi-reference electrode relative to a stable Os-complex (see Fig. S4 in the ESM), clearly confirming that there is no significant shift in the reference potential during the SECCM area scan. Subtraction of the BDD electrode contribution yields the intrinsic $\mathrm{PtNi}_{2}$ activity response, yet since the number of NPs at each spot is unknown, it cannot be quantified, which denotes a presently existing limitation of this technique for such small particle sizes.

To assess the meaning of the data generated with the SECCM experiment, we were looking for a technique which provides an estimation of the current from a single NP for quantification of the SECCM data. For this purpose, we used focused ion beam (FIB) processed carbon nanoelectrodes since they can be analysed by means of TEM using a specifically developed TEM holder. SEM-FIB processing allows us to create a sharp and flat nanoelectrode surface, which is ideal for this goal. Typical FIB processing would cause the nanoelectrode surface to be in a $90^{\circ}$ angle relative to the TEM focus plane though, which is why we performed cutting of the electrode in a $45^{\circ}$ angle (Figs. 3(a)-3(c)). For immobilisation of $\mathrm{PtNi}_{2} \mathrm{NPs}$ of the same batch, we used potential-assisted immobilisation making use of the Brownian motion of NPs in the suspension medium [13]. Thermal annealing caused activation of the particles by removal of the oleylamine capping layers [45] and an increased current compared to the blank electrode was observed (Fig. 3(g)). Subtraction of the blank electrode current 
(a)

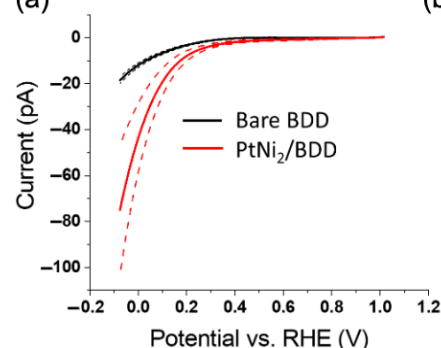

(b)

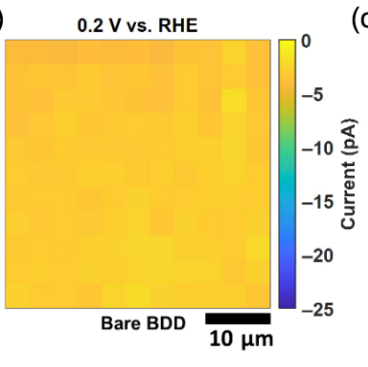

(c)

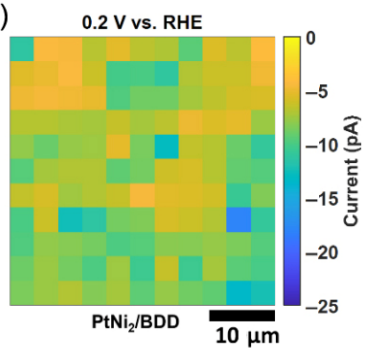

Figure 2 (a) ORR LSVs in $0.1 \mathrm{M} \mathrm{KOH}$ obtained via an SECCM scan on a boron-doped diamond electrode. The KOH was not purged with oxygen to ensure optimal stability of oxygen concentration under ambient air conditions. In black, the blank electrode is shown, in red the combined response after $\mathrm{PtNi}_{2} \mathrm{NP}$ immobilisation and removal of the capping layer is shown. The straight lines represent the average responses and the dashed lines show their standard deviations from 121 independent measurements. ((b) and (c)) Colour plots showing the obtained current at each spot at $0.2 \mathrm{~V}$ vs. reversible hydrogen electrode (RHE) of the bare electrode and the surface after immobilisation of NPs, respectively. The size of the SECCM capillary is $\sim 800 \mathrm{~nm}$ and the scan covers a $40 \mu \mathrm{m} \times 40 \mu \mathrm{m}$ area with a hopping distance of $4 \mu \mathrm{m}$.

(a)

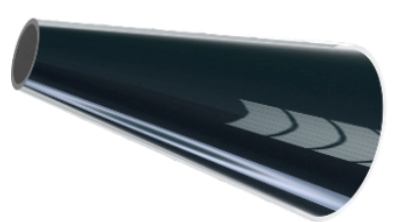

(d)

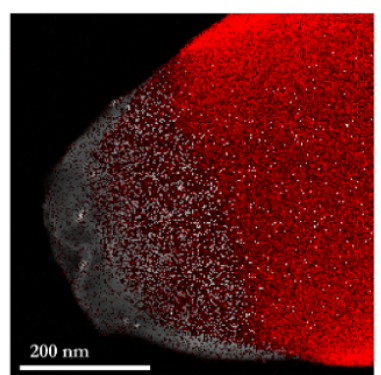

(b)

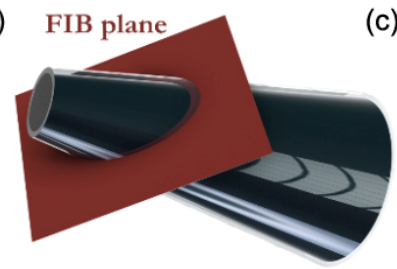

(e)

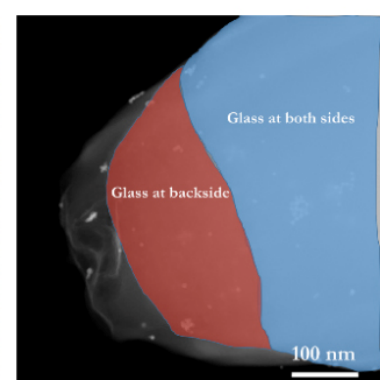

(c)

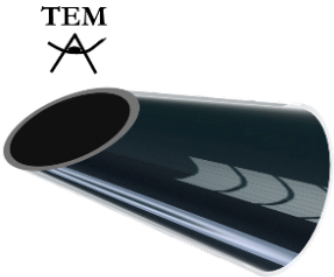

(f)

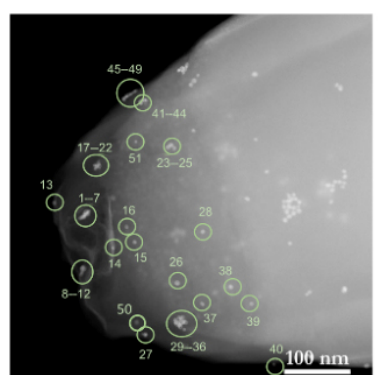

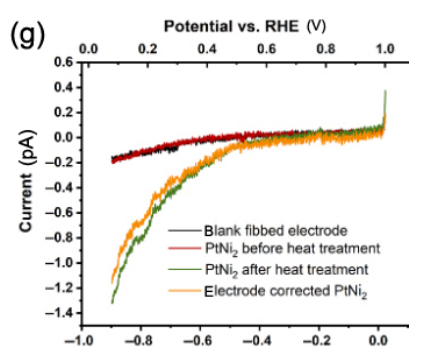

(h)

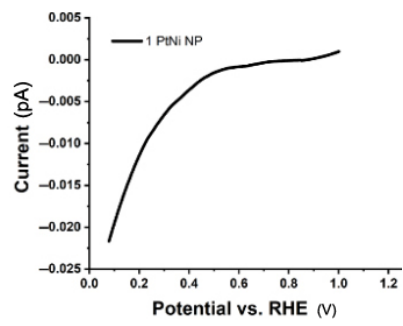

Figure 3 (a)-(c) Schematic illustration of the FIB process of a carbon nanoelectrode with a cutting angle of $45^{\circ}$. TEM beam orientation is shown in (c).

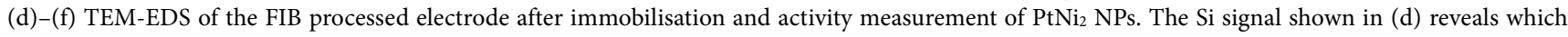
NPs are immobilised on top of the glass insulation and thus, not in contact with the electrode (back and front sides of the capillary are indicated in (e)), which allows counting of current contributing NPs (f). $(\mathrm{g}$ ) and (h) Electrochemical ORR measurements in $0.1 \mathrm{M}$ KOH under ambient air oxygen concentration of the FIB processed electrode.

yields the current, which can be fully assigned to the catalytic response invoked by the $\mathrm{PtNi}_{2} \mathrm{NPs}$. For quantification of their number, the electrode was transferred to a TEM. Electron dispersive X-ray spectroscopy (EDS) analysis revealed the cut edge of the insulating glass and thus, the exposed area of the electrode (Fig. 3(d)). The dense Si area indicates a complete cylindrical sealing of the glass capillary, whereas the low intensity region marks the backside which is still covered with glass due to the $45^{\circ}$ FIB angle. Adjustment of the beam focus clearly separates two groups of particles. One group becomes sharp at a focus in direction of the electron source and vice versa. Hence, one can simply distinguish between the particles which are attached at the glass at the backside and are not in electrical contact to the electrode surface and the frontside particles, which are immobilised at the electrode surface and contribute to the catalytic current. Counting of the particles yields a number of 51 (Fig. 3(f)), implying that the measured current can be divided by 51 to obtain the current a single NP would generate (Fig. 2(h)).

This knowledge can be used to quantitatively analyse the SECCM data. By division of the current at high overpotential of each SECCM spot by the current referring to one particle as determined with the fibbed electrode approach, the number of particles at each spot can be estimated and the corresponding results are displayed in Fig. 4(a). Since small errors in the single NP curve translate to much bigger errors in the determination of the number of particles for the SECCM measurement, these numbers should not be interpreted as exact, but they reflect the approximate range, which is between 80 and 1,300 NPs for this study in a Gaussian like distribution centred around 300-400 NPs (Fig. 4(b)). This range is suitable for providing statistical averaging of the particle size distribution and thus, yields realistic averaged information about the particle ensemble. This would allow transfer of the information on bigger scales as well. However, the intrinsic activity would only be obtained when the particles act as independent units and do not interact with each other unlike for instance agglomerates, where a fraction of the surface is blocked by the neighbouring particles. With an average diameter of the wetted surface area for each spot of about $800 \mathrm{~nm}$ (see Fig. S3 in the ESM), the possible number of NPs exhibiting a diameter of $9 \mathrm{~nm}$ can be calculated until a full monolayer coverage is reached for 6,183 particles, which is way higher than the approximate number of particles even at the spot of highest current. This implies that the chosen NP concentration for drop-casting was a suitable compromise between enabling enough dilution for having less than monolayer coverage as necessary for intrinsic activity analysis while having sufficient coverage to achieve an acceptable signal to noise 

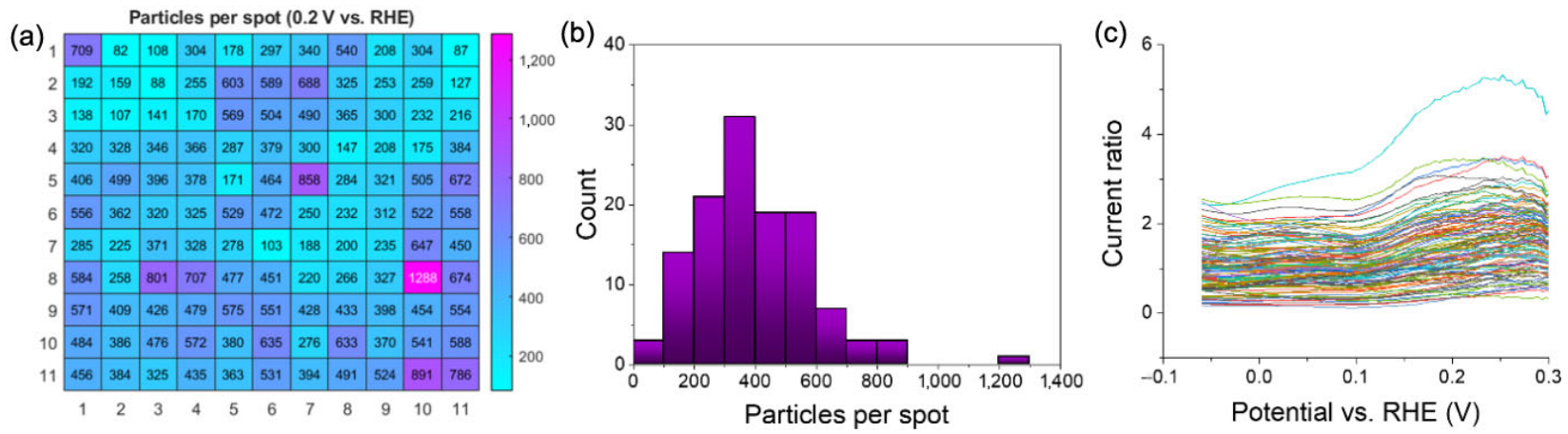

Figure 4 (a) Information about the approximate number of $\mathrm{PtNi}_{2} \mathrm{NPs}$ at each spot of the SECCM measurement based on the knowledge of a single NP current. (b) Histogram of the NP number distribution of all 121 SECCM measurements. (c) Current ratios at the kinetic region for all spots relative to a spot with intermediate NP number for evaluation of linear scaling of different NP numbers.

ratio and statistical representation of the NP population. To further confirm the statistical representation, all blank BDDelectrode corrected LSV curves should scale with a constant factor throughout the whole kinetic region with this factor representing the ratio of the different NP numbers in the different measured surface areas. Tafel analysis confirms kinetic behaviour according to the Butler-Volmer equation without any influence of mass transport effects for the entire potential range, enabled by enhanced hemispherical diffusion towards the NPs (see Fig. S5(c) in the ESM). In Fig. 4(c), a representative current ratio of all curves relative to an arbitrary spot with intermediate number of particles is shown. Whereas all lines confirm a rather constant current ratio and thus, NP ratio quite well, the accuracy becomes even higher for smaller current ratios (comparing LSVs of higher NP numbers) and higher overpotentials. This can be rationalised by the higher probability of statistical representation of the NP population with averaging out the size distribution and the reduced effect of noise at higher overpotentials.

\section{Conclusion}

SECCM provides several advantages to obtain intrinsic activity information averaged for an entire NP size distribution, which is the goal for verification of theoretically predicted activity properties. However, for catalytically relevant small particle sizes, the determination of the NP number and thus, their surface becomes a challenge. In general, we highlighted why electrode contribution subtraction becomes important especially for small NP sizes, how the reference electrode shift must be ruled out and can be monitored and how the linear scaling between different NP numbers can be evaluated. Quantification remains the biggest challenge and it is generally unknown whether many multilayers are present, a very small coverage of isolated NPs or something in between. We demonstrated that this information can be gathered using a complementary "ruler" technique, which alone enables to investigate the activity current profile of a single NP. Furthermore, its extrapolation confirms that also statistical analysis of intrinsic activity representative for the NP population can be achieved with SECCM, where also with very small NPs the signal to noise ratio is sufficient to investigate isolated NPs. Hence, both techniques allow to accomplish the challenging search for intrinsic properties, also for very small NP sizes, when the SECCM substrate does not fit into a TEM or the wetted area of each spot cannot be identified.

\section{Acknowledgements}

This project has received funding from the European Research
Council (ERC) under the European Union's Horizon 2020 research and innovation programme (grant agreement CasCat [833408]) as well as from the European Union's Horizon 2020 research and innovation program under the Marie Skłodowska-Curie MSCA-ITN Single-Entity Nanoelectrochemistry, Sentinel [812398]. S. S. and C. A. acknowledge the Deutsche Forschungsgemeinschaft (DFG, German Research Foundation) within the project [440951282]. X. X. C. acknowledges financial support from the Liaoning BaiQianWan Talents Program, China (No. 2019B042), and the Excellent Young Scientific and Technological Talents Project of Educational Department of Liaoning Province, China (No. 2020LNQN07). We acknowledge Prof. Patrick Unwin from the University of Warwick for providing the initial control software (WEC-SPM) for our SECCM experiments.

Funding note: Open Access funding enabled and organized by Projekt DEAL.

Electronic Supplementary Material: Supplementary material (experimental details, characterisation of $\mathrm{PtNi}_{2}$ nanoparticles, etc) is available in the online version of this article at https://doi.org/10.1007/s12274-021-3702-7.

Open Access This article is licensed under a Creative Commons Attribution 4.0 International License, which permits use, sharing, adaptation, distribution and reproduction in any medium or format, as long as you give appropriate credit to the original author(s) and the source, provide a link to the Creative Commons licence, and indicate if changes were made.

The images or other third party material in this article are included in the article's Creative Commons licence, unless indicated otherwise in a credit line to the material. If material is not included in the article's Creative Commons licence and your intended use is not permitted by statutory regulation or exceeds the permitted use, you will need to obtain permission directly from the copyright holder.

To view a copy of this licence, visit http://creativecommons.org/licenses/by/4.0/.

\section{References}

[1] Nesselberger, M.; Ashton, S.; Meier, J. C.; Katsounaros, I.; Mayrhofer, K. J. J.; Arenz, M. The particle size effect on the oxygen reduction reaction activity of $\mathrm{Pt}$ catalysts: Influence of electrolyte and relation to single crystal models. J. Am. Chem. Soc. 2011, 133, 17428-17433.

[2] Hall, A. S.; Yoon, Y.; Wuttig, A.; Surendranath, Y. Mesostructureinduced selectivity in $\mathrm{CO}_{2}$ reduction catalysis. J. Am. Chem. Soc. 2015, 137, 14834-14837. 
[3] Song, F.; Hu, X. L. Exfoliation of layered double hydroxides for enhanced oxygen evolution catalysis. Nat. Commun. 2014, 5, 4477.

[4] Chen, C.; Kang, Y. J.; Huo, Z. Y.; Zhu, Z. W.; Huang, W. Y.; Xin, H. L.; Snyder, J. D.; Li, D. G.; Herron, J. A.; Mavrikakis, M. et al. Highly crystalline multimetallic nanoframes with three-dimensional electrocatalytic surfaces. Science 2014, 343, 1339-1343.

[5] Sasikumar, G.; Ihm, J.W.; Ryu, H. Dependence of optimum Nafion content in catalyst layer on platinum loading. J. Power Sources 2004, 132, 11-17.

[6] Batchelor, T. A. A.; Pedersen, J. K.; Winther, S. H.; Castelli, I. E.; Jacobsen, K. W.; Rossmeisl, J. High-entropy alloys as a discovery platform for electrocatalysis. Joule 2019, 3, 834-845.

[7] Nørskov, J. K.; Rossmeisl, J.; Logadottir, A.; Lindqvist, L.; Kitchin, J. R.; Bligaard, T.; Jónsson, H. Origin of the overpotential for oxygen reduction at a fuel-cell cathode. J. Phys. Chem. B 2004, 108, 17886-17892.

[8] Behrens, M.; Studt, F.; Kasatkin, I.; Kühl, S.; Hävecker, M.; Abild-Pedersen, F.; Zander, S.; Girgsdies, F.; Kurr, P.; Kniep, B. L. et al. The active site of methanol synthesis over $\mathrm{Cu} / \mathrm{ZnO} / \mathrm{Al}_{2} \mathrm{O}_{3}$ industrial catalysts. Science 2012, 336, 893-897.

[9] Guo, D. H.; Shibuya, R.; Akiba, C.; Saji, S.; Kondo, T.; Nakamura, J. Active sites of nitrogen-doped carbon materials for oxygen reduction reaction clarified using model catalysts. Science 2016, 351,361-365.

[10] Chai, G. L.; Qiu, K. P.; Qiao, M.; Titirici, M. M.; Shang, C. X.; Guo, Z. X. Active sites engineering leads to exceptional ORR and OER bifunctionality in P,N Co-doped graphene frameworks. Energy Environ. Sci. 2017, 10, 1186-1195.

[11] Batchellor, A. S.; Boettcher, S. W. Pulse-electrodeposited Ni-Fe (oxy)hydroxide oxygen evolution electrocatalysts with high geometric and intrinsic activities at large mass loadings. ACS Catal. 2015, 5, 6680-6689.

[12] Pope, M. A.; Punckt, C.; Aksay, I. A. Intrinsic capacitance and redox activity of functionalized graphene sheets. J. Phys. Chem. C 2011, 115, 20326-20334.

[13] Löffler, T.; Wilde, P.; Öhl, D.; Chen, Y. T.; Tschulik, K.; Schuhmann, W. Evaluation of the intrinsic catalytic activity of nanoparticles without prior knowledge of the mass loading. Faraday Discuss. 2018, 210, 317-332.

[14] Wang, Y.; Laborda, E.; Plowman, B. J.; Tschulik, K.; Ward, K. R.; Palgrave, R. G.; Damm, C.; Compton, R. G. The strong catalytic effect of $\mathrm{Pb}$ (II) on the oxygen reduction reaction on $5 \mathrm{~nm}$ gold nanoparticles. Phys. Chem. Chem. Phys. 2014, 16, 3200-3208.

[15] Wang, Y.; Laborda, E.; Tschulik, K.; Damm, C.; Molina, A.; Compton, R. G. Strong negative nanocatalysis: Oxygen reduction and hydrogen evolution at very small $(2 \mathrm{~nm})$ gold nanoparticles. Nanoscale 2014, 6, 11024-11030.

[16] Wilde, P.; Barwe, S.; Andronescu, C.; Schuhmann, W.; Ventosa, E. High resolution, binder-free investigation of the intrinsic activity of immobilized NiFe LDH nanoparticles on etched carbon nanoelectrodes. Nano Res. 2018, 11, 6034-6044.

[17] Lai, S. C. S.; Dudin, P. V.; Macpherson, J. V.; Unwin, P. R. Visualizing zeptomole (electro)catalysis at single nanoparticles within an ensemble. J. Am. Chem. Soc. 2011, 133, 10744-10747.

[18] Li, Y. X.; Cox, J. T.; Zhang, B. Electrochemical responses and electrocatalysis at single Au nanoparticles. J. Am. Chem. Soc. 2010, 132, 3047-3054.

[19] Kang, M.; Perry, D.; Bentley, C. L.; West, G.; Page, A.; Unwin, P. R. Simultaneous topography and reaction flux mapping at and around electrocatalytic nanoparticles. ACS Nano 2017, 11, 9525-9535.

[20] Ly, L. S. Y.; Batchelor-McAuley, C.; Tschulik, K.; Kätelhön, E.; Compton, R. G. A critical evaluation of the interpretation of electrocatalytic nanoimpacts. J. Phys. Chem. C 2014, 118, 1775617763.

[21] Ebejer, N.; Schnippering, M.; Colburn, A. W.; Edwards, M. A.; Unwin, P. R. Localized high resolution electrochemistry and multifunctional imaging: Scanning electrochemical cell microscopy. Anal. Chem. 2010, 82, 9141-9145.

[22] Wahab, O. J.; Kang, M.; Unwin, P. R. Scanning electrochemical cell microscopy: A natural technique for single entity electrochemistry. Curr. Opin. Electrochem. 2020, 22, 120-128.

[23] Snowden, M. E.; Güell, A. G.; Lai, S. C. S.; McKelvey, K.; Ebejer, N.; O'Connell, M. A.; Colburn, A. W.; Unwin, P. R. Scanning electrochemical cell microscopy: Theory and experiment for quantitative high resolution spatially-resolved voltammetry and simultaneous ion-conductance measurements. Anal. Chem. 2012, 84, 2483-2491.

[24] Güell, A. G.; Cuharuc, A. S.; Kim, Y. R.; Zhang, G. H.; Tan, S. Y.; Ebejer, N.; Unwin, P. R. Redox-dependent spatially resolved electrochemistry at graphene and graphite step edges. ACS Nano 2015, 9, 3558-3571.

[25] Aaronson, B. D. B.; Chen, C. H.; Li, H. J.; Koper, M. T. M.; Lai, S. C. S.; Unwin, P. R. Pseudo-single-crystal electrochemistry on polycrystalline electrodes: Visualizing activity at grains and grain boundaries on platinum for the $\mathrm{Fe}^{2+} / \mathrm{Fe}^{3+}$ redox reaction. J. Am . Chem. Soc. 2013, 135, 3873-3880.

[26] Bentley, C. L.; Andronescu, C.; Smialkowski, M.; Kang, M.; Tarnev, T.; Marler, B.; Unwin, P. R.; Apfel, U. P.; Schuhmann, W. Local surface structure and composition control the hydrogen evolution reaction on Iron Nickel sulfides. Angew. Chem., Int. Ed. 2018, 57, 4093-4097.

[27] Chen, C. H.; Jacobse, L.; McKelvey, K.; Lai, S. C. S.; Koper, M. T. M.; Unwin, P. R. Voltammetric scanning electrochemical cell microscopy: Dynamic imaging of hydrazine electro-oxidation on platinum electrodes. Anal. Chem. 2015, 87, 5782-5789.

[28] Tao, B. L.; Unwin, P. R.; Bentley, C. L. Nanoscale variations in the electrocatalytic activity of layered transition-metal dichalcogenides. J. Phys. Chem. C 2020, 124, 789-798.

[29] Bentley, C. L.; Agoston, R.; Tao, B. L.; Walker, M.; Xu, X. D.; O'Mullane, A. P.; Unwin, P. R. Correlating the local electrocatalytic activity of amorphous molybdenum sulfide thin films with microscopic composition, structure, and porosity. ACS Appl. Mater. Interfaces 2020, 12, 44307-44316.

[30] Oseland, E. E.; Ayres, Z. J.; Basile, A.; Haddleton, D. M.; Wilson, P.; Unwin, P. R. Surface patterning of polyacrylamide gel using scanning electrochemical cell microscopy (SECCM). Chem. Commun. 2016, 52, 9929-9932.

[31] Gao, R.; Edwards, M. A.; Qiu, Y. H.; Barman, K.; White, H. S. Visualization of hydrogen evolution at individual platinum nanoparticles at a buried interface. J. Am. Chem. Soc. 2020, 142, 8890-8896.

[32] Choi, M.; Siepser, N. P.; Jeong, S.; Wang, Y.; Jagdale, G.; Ye, X. C.; Baker, L. A. Probing single-particle electrocatalytic activity at facet-controlled gold nanocrystals. Nano Lett. 2020, 20, 1233-1239.

[33] Chen, C. H.; Ravenhill, E. R.; Momotenko, D.; Kim, Y. R.; Lai, S. C. S.; Unwin, P. R. Impact of surface chemistry on nanoparticleelectrode interactions in the electrochemical detection of nanoparticle collisions. Langmuir 2015, 31, 11932-11942.

[34] Kleijn, S. E. F.; Lai, S. C. S.; Miller, T. S.; Yanson, A. I.; Koper, M. T. M.; Unwin, P. R. Landing and catalytic characterization of individual nanoparticles on electrode surfaces. J. Am. Chem. Soc. 2012, 134, 18558-18561.

[35] Saha, P.; Hill, J. W.; Walmsley, J. D.; Hill, C. M. Probing electrocatalysis at individual Au nanorods via correlated optical and electrochemical measurements. Anal. Chem. 2018, 90, 1283212839 .

[36] Ornelas, I. M.; Unwin, P. R.; Bentley, C. L. High-throughput correlative electrochemistry-microscopy at a transmission electron microscopy grid electrode. Anal. Chem. 2019, 91, 14854-14859.

[37] Ustarroz, J.; Ornelas, I. M.; Zhang, G. H.; Perry, D.; Kang, M.; Bentley, C. L.; Walker, M.; Unwin, P. R. Mobility and poisoning of mass-selected platinum nanoclusters during the oxygen reduction reaction. ACS Catal. 2018, 8, 6775-6790.

[38] Chen, C. H.; Meadows, K. E.; Cuharuc, A.; Lai, S. C. S.; Unwin, P. R. High resolution mapping of oxygen reduction reaction kinetics at polycrystalline platinum electrodes. Phys. Chem. Chem. Phys. 2014, 16, 18545-18552.

[39] Bentley, C. L.; Kang, M.; Unwin, P. R. Scanning electrochemical 
cell microscopy (SECCM) in aprotic solvents: Practical considerations and applications. Anal. Chem. 2020, 92, 11673-11680.

[40] Hill, J. W.; Fu, Z. G.; Tian, J. F.; Hill, C. M. Locally engineering and interrogating the photoelectrochemical behavior of defects in transition metal dichalcogenides. J. Phys. Chem. C 2020, 124, 17141-17149.

[41] Daviddi, E.; Shkirskiy, V.; Kirkman, P. M.; Robin, M. P.; Bentley, C. L.; Unwin, P. R. Nanoscale electrochemistry in a copper/aqueous/oil three-phase system: surface structure-activity-corrosion potential relationships. Chem. Sci. 2021, 12, 3055-3069.

[42] Li, Y. J.; Morel, A.; Gallant, D.; Mauzeroll, J. Oil-immersed scanning micropipette contact method enabling long-term corrosion mapping. Anal. Chem. 2020, 92, 12415-12422.
[43] Yang, N. J.; Yu, S. Y.; Macpherson, J. V.; Einaga, Y.; Zhao, H. Y.; Zhao, G. H.; Swain, G. M.; Jiang, X. Conductive diamond: synthesis, properties, and electrochemical applications. Chem. Soc. Rev. 2019, 48, 157-204.

[44] Yu, Y. S.; Yang, W. W.; Sun, X. L.; Zhu, W. L.; Li, X. Z.; Sellmyer, D. J.; Sun, S. H. Monodisperse MPt (M=Fe, Co, Ni, Cu, Zn) nanoparticles prepared from a facile oleylamine reduction of metal salts. Nano Lett. 2014, 14, 2778-2782.

[45] Benedetti, T. M.; Andronescu, C.; Cheong, S.; Wilde, P.; Wordsworth, J.; Kientz, M.; Tilley, R. D.; Schuhmann, W.; Gooding, J. J. Electrocatalytic nanoparticles that mimic the three-dimensional geometric architecture of enzymes: Nanozymes. J. Am. Chem. Soc. 2018, 140, 13449-13455. 